\title{
Coronary surgery following prior pneumonectomy -A challenging clinical scenario with excellent results
}

\author{
Stefanie Reutter, Oliver Liakopoulos, Yeong-Hoon Choi, Thorsten Wahlers, Thorsten Wittwer ${ }^{*}$ \\ Department of Cardiothoracic Surgery, Heart Center, University Hospital of Cologne, Kolner, Germany; \\ *Corresponding Author: th.wittwer-md@t-online.de
}

Received 29 November 2012; revised 25 January 2013; accepted 6 January 2013

\begin{abstract}
Coronary artery bypass grafting (CABG) in patients with prior pneumonectomy is challenging because of the decreased pulmonary function and the anatomical alterations of the thoracic cavity. Due to extended morbidity and mortality in this special patient cohort, only very few reports in the world literature focus on this topic. Overall, no general recommendations exist with regard to the optimal management of this continuously growing patient cohort. We present our experience with another successful case.
\end{abstract}

Keywords: Coronary Surgery; Previous Pneumonectomy

\section{INTRODUCTION}

Coronary artery bypass grafting (CABG) in patients with prior pneumonectomy represents a significant surgical challenge as this modality results in hyperinflation of the contralateral lung with shifting of the heart and mediastinum and elevation of the diaphragma on the operated side [1]. Furthermore, operative trauma and radiation frequently produce severe adhesions that may additionally complicate subsequent cardiac surgery. In a clinical review of only 19 internationally published cases up to 2007, Stoller et al. [1] reported a postoperative mortality rate of $16 \%$ and emphasized the importance of ongoing summarizing the aggregate experience with this high risk patients. As still no general recommendations exist with regard to the optimal management of patients with prior pneumonectomy scheduled for cardiac surgery, our latest experience adds another successful case of to the current literature.

\section{CLINICAL SUMMARY}

A 61-year-old male patient with a history of left pneumonectomy and postoperative irradiation for squamous cell lung carcinoma (pT3 pN0 cM0) 28 years ago was referred to our hospital with an acute STEMI. Coronary angiography revealed severe three vessel disease with intact LV function, pulmonary evaluation showed a combined obstructive and restrictive pattern $\left(\mathrm{FEV}_{1} 1.36\right.$ L; 42\% predicted; FVC 1.54 L; 36\% predicted). Blood gases showed normal $\mathrm{pH}$, mild hypercapnia $\left(\mathrm{paCO}_{2}: 47\right.$ $\mathrm{mmHg})$ and hypoxemia $\left(\mathrm{paO}_{2}: 68 \mathrm{mmHg}\right)$. Computed tomography (CT) presented a moderate shift of the heart and the great vessels into the left hemithorax with an overinflated right lung crossing the midline (Figure 1). Although some authors suggest a left thoracotomy as a favourable surgical approach [2,3], we decided to approach the heart via a median sternotomy due to only moderate shifting of the heart and the need for bypass grafting of both the left and the right coronary artery. Sternotomy was performed during apnea to protect the right lung that crossed the sternal midline. After careful dissection of severe adhesions with utmost care to protection of the right lung, preparation of the left ITA revealed complete obliteration due to previous irradiation. With respect to the integrity of the right lung, we avoided additional RITA harvesting and the patient underwent uneventful on-pump CABG with single vein grafts of

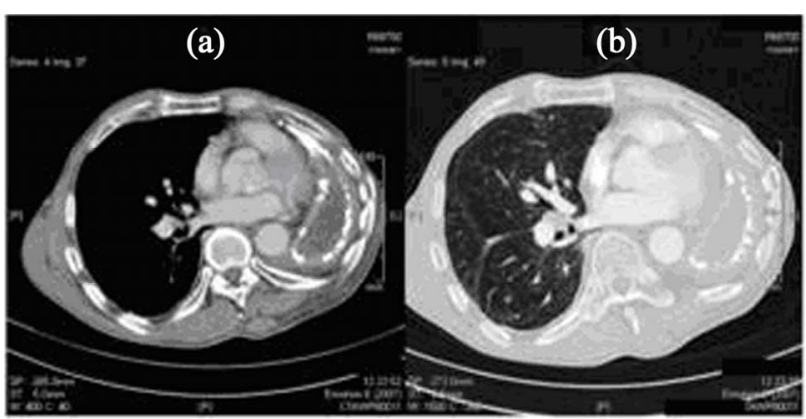

Figure 1. Preoperative computed tomography of the chest shows a moderate shift of the heart and the great vessels into the left hemithorax (a) with a compensatory overinflation of the right lung crossing the midline (b). 
LAD, RCx and RCA using antegrade blood cardioplegia at $32^{\circ} \mathrm{C}$. Peri- and postoperative fluid management was guided using accurate estimation of left ventricular preload by measuring the pulmonary capillary wedge pressure. During the operation, the pulmonary artery, pulmonary capillary wedge, and central venous pressure were measured as 36/18 mmHg, $19 \mathrm{mmHg}$, and 12 $\mathrm{mmHg}$, respectively. Early postoperative hemodynamic values were rather unchanged.

Following meticulous pulmonary weaning, extubation was performed after 36 hours without problems. Postoperative blood gases were similar to preoperative values. After an uncomplicated postoperative course, the patient was discharged on the ninth postoperative day and remains asymptomatic for more than 4 months now.

\section{DISCUSSION}

CABG in patients with prior pneumonectomy is challenging due to the decreased pulmonary function and the anatomical alterations of the thoracic cavity. In a review of the sparse available literature by Stoller et al., the 30-day mortality of patients undergoing CABG after pneumonectomy was $16 \%$. Postoperative complications were reported in $50 \%$ of the cases and were mainly due to respiratory insufficiency necessitating reintubation [1]. Because of these limitations, prior pneumonectomy requires several special surgical considerations regarding exposure, prevention of diaphragmatic dysfunction, and choice of bypass conduits in CABG procedures. Regarding exposure, previous left pneumonectomy causes a shift of mediastinal structures into the left hemithorax and access to the circumflex coronaries could become difficult through median sternotomy. Thus, some authors described a left thoracotomy as a favourable surgical approach [2,3]. In our case, we based the surgical approach on a preoperative CT which showed only slight shifting of the heart with potentially easy access to all coronary arteries via median sternotomy.

The use of the internal thoracic artery (ITA) versus saphenous vein (SV) as a bypass conduit is also a subject of considerable debate. As harvesting of the right ITA in patients with previous left pneumonectomy may result in significantly decreased postoperative pulmonary function $[1,4]$ with risk of injury to the phrenic nerve resulting in persistent diaphragmatic paralysis [5], it is prudent in our experience to avoid ITA harvest on the side of the intact lung to prevent serious consequences of phrenic nerve injury. Because of these considerations, we exclusively used vein grafts in our patient who had undergone left pneumonectomy as the left ITA was obliterated due to previous irradiation.

As a conclusion, this latest experience confirms that coronary surgery following previous pneumonectomy still represents a challenging clinical scenario. Based on the little available data published in the literature, we believe that with utmost attention to the specific features of the perioperative management, this approach can be performed with excellent results in specialized centers and is therefore justified in appropriately selected and managed patients.

\section{REFERENCES}

[1] Stoller, J.K., Blackstone, E., Pettersson, G. and Mihaljevic, T. (2007) Coronary artery bypass graft and/or valvular operations following prior pneumonectomy: Report of four new patients and review of the literature. Chest, 132, 295-301. doi:10.1378/chest.06-2545

[2] Ushijima, T., Kikuchi, Y., Ikeda, C., Takata, M., Yamamoto, Y. and Watanabe, G. (2011) Totally arterial offpump coronary artery bypass grafting after pneumonictomy. Annals of Thoracic and Cardiovascular Surgery, 17, 320-322. doi:10.5761/atcs.cr.10.01534

[3] Zhao, B.Q., Chen, R.K. and Song, J.P. (2008) Coronary artery bypass grafting after pneumonectomy. Texas Heart Institute Journal, 35, 470-471.

[4] Vargas, F.S., terra-Filho, M., Hueb, W., Teixeira, L.R., Cukier, A. and Light, R.W. (1997) Pulmonary function after coronary artery bypass surgery. Respiratory Medicine, 91, 629-633. doi:10.1016/S0954-6111(97)90010-X

[5] Efthimiou, J., Butler, J., Woodham, C., Benson, M.K. and Westaby, S. (1991) Diaphragm paralysis following cardiac surgery: Role of phenic nerve cold injury. The Annals of Thoracic Surgery, 52, 1005-1008. doi:10.1016/0003-4975(91)91268-Z 